MATHEMATICS OF COMPUTATION

Volume 80, Number 275, July 2011, Pages 1849-1864

S $0025-5718(2011) 02445-2$

Article electronically published on February 25, 2011

\title{
ON THE INTEGERS OF THE FORM $p^{2}+b^{2}+2^{n}$ AND $b_{1}^{2}+b_{2}^{2}+2^{n^{2}}$
}

\author{
HAO PAN AND WEI ZHANG
}

\begin{abstract}
We prove that the sumset $\left\{p^{2}+b^{2}+2^{n}: p\right.$ is prime and $\left.b, n \in \mathbb{N}\right\}$ has positive lower density. We also construct a residue class with an odd modulus that contains no integer of the form $p^{2}+b^{2}+2^{n}$. Similar results are established for the sumset $\left\{b_{1}^{2}+b_{2}^{2}+2^{n^{2}}: b_{1}, b_{2}, n \in \mathbb{N}\right\}$.
\end{abstract}

\section{INTRODUCTION}

Let $\mathcal{P}$ denote the set of all primes. In 1934, Romanoff [25] proved that the sumset

$$
\mathcal{S}_{1}=\left\{p+2^{n}: p \in \mathcal{P}, n \in \mathbb{N}\right\}
$$

has positive lower density. Subsequently, van der Corput 13 proved that the complement of $\mathcal{S}_{1}$, i.e., $\mathbb{N} \backslash \mathcal{S}_{1}$, also has positive lower density. In fact, Erdős [14] showed that every positive integer $n$ with $n \equiv 7629217(\bmod 11184810)$ is not of the form $p+2^{n}$ with $p \in \mathcal{P}$ and $n \in \mathbb{N}$. The key ingredient of Erdös's proof is to find a finite set of residue classes with distinct moduli, which covers all integers. Over the years, Erdős's idea has been greatly extended [11, 10, 3, 26, 4, 5, 27, 28, 6, 30, 7, 21, 8, 9, 29.

In 1999, with the help of Brüdern and Fouvry's estimates on sums of squares [2], Liu, Liu and Zhan [22] proved a Romanoff-type result:

The sumset

$$
\mathcal{S}_{2}=\left\{p_{1}^{2}+p_{2}^{2}+2^{n_{1}}+2^{n_{2}}: p_{1}, p_{2} \in \mathcal{P}, n_{1}, n_{2} \in \mathbb{N}\right\}
$$

has positive lower density.

The key part of their proof is the following lemma:

Lemma 1.1. For $1 \leqslant m \leqslant N$,

$$
\mid\left\{p_{1}^{2}+p_{2}^{2}-p_{3}^{2}-p_{4}^{2}=m: p_{i} \in \mathcal{P}, p_{i}^{2} \leqslant N\right\} \ll \mathfrak{S}_{-}(m) \frac{N}{(\log N)^{4}},
$$

where $\mathfrak{S}_{-}$will be introduced in Section 2.

Received by the editor May 23, 2009 and, in revised form, April 25, 2010

2010 Mathematics Subject Classification. Primary 11P32; Secondary 11A07, 11B05, 11B25, $11 \mathrm{~N} 36$.

Key words and phrases. Positive lower density, arithmetical progression, prime, square, power of 2 .

The first author is supported by the National Natural Science Foundation of China (Grant No. 10771135 and 10901078).

(C)2011 American Mathematical Society 
In the other direction, recently Crocker [12] proved that there exist infinitely many positive integers not representable as the sum of two squares and two (or fewer) powers of 2.

By the prime number theorem, clearly,

$$
\left|\left\{p^{2}+b^{2} \leqslant N: p \in \mathcal{P}, b \in \mathbb{N}\right\}\right| \leqslant|\mathcal{P} \cap[0, \sqrt{N}]| \cdot|\mathbb{N} \cap[0, \sqrt{N}]| \ll \frac{N}{\log N} .
$$

On the other hand, in 24, Rieger proved that

$$
\left|\left\{p^{2}+b^{2} \leqslant N: p \in \mathcal{P}, b \in \mathbb{N}\right\}\right| \gg \frac{N}{\log N} .
$$

Motivated by all of these results, in the present paper, we shall study the sumset

$$
\mathcal{S}_{3}=\left\{p^{2}+b^{2}+2^{n}: p \in \mathcal{P}, b, n \in \mathbb{N}\right\} .
$$

First, we have the following Romanoff-type result.

Theorem 1.1. The set $\mathcal{S}_{3}$ has positive lower density.

Next, we need to say something about the complement of $\mathcal{S}_{3}$. It is not difficult to see that almost all integers in $\mathcal{S}_{3}$ are of the form $4 k+1$ or $8 k+2$. However, we shall prove that

Theorem 1.2. There exists a residue class with an odd modulus that contains no integer of the form $p^{2}+b^{2}+2^{n}$ with $p \in \mathcal{P}$ and $b, n \in \mathbb{N}$.

Since the modulus in Theorem 1.2 is odd, by the Chinese remainder theorem, clearly both the sets

$$
\left\{x \in \mathbb{N}: x \equiv 1(\bmod 4), x \notin \mathcal{S}_{3}\right\}
$$

and

$$
\left\{x \in \mathbb{N}: x \equiv 2(\bmod 8), x \notin \mathcal{S}_{3}\right\}
$$

have positive lower densities.

Furthermore, we also have a similar result on the integers not of the form $p^{2}+$ $b^{2}-2^{n}$.

Theorem 1.3. There exists a residue class with an odd modulus that contains no integer of the form $p^{2}+b^{2}-2^{n}$ with $p \in \mathcal{P}$ and $b, n \in \mathbb{N}$.

In fact, as we shall see later, our proof can provide the explicit values of the residues and moduli described in Theorems 1.2 and 1.3. although these values are too large to write down here.

A well-known result due to Landau [20] asserts that

$$
\left\{b_{1}^{2}+b_{2}^{2} \leqslant N: b_{1}, b_{2} \in \mathbb{N}\right\}=\frac{K N}{\sqrt{\log N}}(1+o(1))
$$

where

$$
K=\frac{1}{\sqrt{2}} \prod_{\substack{p \in \mathcal{P} \\ p \equiv 3(\bmod 4)}}\left(1-\frac{1}{p^{2}}\right)^{-1 / 2}=0.764223653 \ldots
$$

Obviously,

$$
\left\{n \in \mathbb{N}: 2^{n^{2}} \leqslant N\right\} \ll \sqrt{\log N} .
$$

These facts suggest that we can obtain the following results. 
Theorem 1.4. The sumset

$$
\mathcal{S}_{4}=\left\{b_{1}^{2}+b_{2}^{2}+2^{n^{2}}: b_{1}, b_{2}, n \in \mathbb{N}\right\}
$$

has positive lower density. Conversely, there also exists a residue class with an odd modulus that contains no integer of the form $b_{1}^{2}+b_{2}^{2}+2^{n^{2}}$.

As to the latter conclusion of the theorem, we actually establish a stronger result (see Theorem 4.1 below).

The proofs of Theorem 1.1 and the former conclusion of Theorem 1.4 are applications of the sieve method. We shall construct a suitable cover of $\mathbb{Z}$ with odd moduli to prove Theorem 1.2, Theorem 1.3, and the latter assertion of Theorem 1.4. Throughout this paper, the constants implied by $\ll, \gg$ and $O(\cdot)$ will always be absolute.

\section{Proof of Theorem 1.1}

For $\mathbf{d}=\left(d_{1}, d_{2}, d_{3}, d_{4}\right)$ with $\mu(\mathbf{d}):=\mu\left(d_{1}\right) \mu\left(d_{2}\right) \mu\left(d_{3}\right) \mu\left(d_{4}\right) \neq 0$, define

$$
A(m, q, \mathbf{d})=q^{-4} \sum_{\substack{1 \leqslant a \leqslant q \\(a, q)=1}} e(-a m / q) S\left(q, a d_{1}^{2}\right) S\left(q, a d_{4}^{2}\right) S\left(q,-a d_{2}^{2}\right) S\left(q,-a d_{3}^{2}\right)
$$

and

$$
\mathfrak{S}(m, \mathbf{d})=\sum_{q=1}^{\infty} A(m, q, \mathbf{d})
$$

where

$$
S(q, a)=\sum_{x=1}^{q} e\left(a x^{2} / q\right)
$$

and $e(\alpha)=\exp (2 \pi \sqrt{-1} \alpha)$. In particular, we set $\mathfrak{S}_{-}(m)=\mathfrak{S}(m,(1,1,1,1))$ and $\omega(\mathbf{d}, m)=\mathfrak{S}(m, \mathbf{d}) / \mathfrak{S}_{-}(m)$.

For a prime $p$ and a positive integer $m$, if $p \nmid m$, then define

$$
\begin{aligned}
& \omega_{1,0}(p, m)= \begin{cases}p /(p-1), & \text { if }\left(\frac{-m}{p}\right)=1, \\
p /(p+1), & \text { if }\left(\frac{-m}{p}\right)=-1,\end{cases} \\
& \omega_{0,1}(p, m)= \begin{cases}p /(p-1), & \text { if }\left(\frac{m}{p}\right)=1, \\
p /(p+1), & \text { if }\left(\frac{m}{p}\right)=-1,\end{cases}
\end{aligned}
$$

and $\omega_{1,1}(p, m)=p /(p+1)$. Also, if $p^{\beta} \| m$ for some $\beta \geqslant 1$, where $p^{\beta} \| m$ means $p^{\beta} \mid m$ but $p^{\beta+1} \nmid m$, then define

$$
\omega_{1,0}(p, m)=\omega_{0,1}(p, m)=\frac{1+p^{-1}-p^{-\beta}-p^{-\beta-1}}{1+p^{-1}-p^{-\beta-1}-p^{-\beta-2}}
$$

and

$$
\omega_{1,1}(p, m)=\frac{3-p^{-1}-p^{1-\beta}-p^{-\beta}}{1+p^{-1}-p^{-\beta-1}-p^{-\beta-2}} .
$$


Lemma 2.1 (Liu, Liu and Zhan [22, eq. (8.7), Lemmas 8.1 and 8.2]). Suppose that $d_{1}, d_{2}, d_{3}, d_{4}$ are square-free and $\left(d_{1}, d_{4}\right)=\left(d_{2}, d_{3}\right)=1$. Then for $\mathbf{d}=\left(d_{1}, d_{2}, d_{3}, d_{4}\right)$ and positive integer $m$, we have

$$
\omega(\mathbf{d}, m)=\prod_{\substack{p \mid d_{1} d_{2} d_{3} d_{4} \\ p^{u}\left\|d_{1} d_{4}, p^{v}\right\| d_{2} d_{3}}} \omega_{u, v}(p, m),
$$

Let

$$
\mathscr{A}=\left\{\left(x_{1}, x_{2}, x_{3}, x_{4}\right): x_{1}^{2}+x_{4}^{2}=x_{2}^{2}+x_{3}^{2}+m, 1 \leqslant x_{i}^{2} \leqslant N\right\},
$$

and for $\mathbf{d}=\left(d_{1}, d_{2}, d_{3}, d_{4}\right)$, let

$$
\mathscr{A}_{\mathbf{d}}=\left\{\left(x_{1}, x_{2}, x_{3}, x_{4}\right) \in \mathscr{A}: x_{i} \equiv 0\left(\bmod d_{i}\right)\right\} .
$$

Lemma 2.2 (Brüdern and Fouvry [2, Theorem 3], Liu, Liu and Zhan [22, Lemma 9.1]). Let

$$
\left|\mathscr{A}_{\mathbf{d}}\right|=\frac{\omega(\mathbf{d}, m)}{d_{1} d_{2} d_{3} d_{4}} \frac{\pi}{16} \mathfrak{S}_{-}(m) \mathfrak{I}(m / N) N+R(m, N, \mathbf{d}),
$$

where

$$
\Im(\theta)=2 \int_{\max \{0,-\theta\}}^{\min \{1,1-\theta\}} t^{-1 / 2}(1-\theta-t)^{1 / 2} d t,
$$

and the remainder term $R(m, N, \mathbf{d})$ satisfies

$$
\sum_{\substack{d_{1}, d_{2}, d_{3}, d_{4} \leqslant N^{1 / 23} \\ \mathbf{d}=\left(d_{1}, d_{2}, d_{3}, d_{4}\right) \\|\mu(d)|=1}}|R(m, N, \mathbf{d})| \ll_{\epsilon} N^{1-\epsilon}
$$

for sufficiently small $\epsilon>0$.

Since $m$ is always fixed, below we abbreviate $\omega_{u, v}(p, m)$ to $\omega_{u, v}(p)$.

Let $D=N^{1 / 30}$ and $z=N^{1 / 300}$. Define

$$
P(z)=\prod_{\substack{p<z \\ p \text { prime }}} p .
$$

Let

$$
f(k)=\left|\left\{\left(x_{1}, x_{2}, x_{3}, x_{4}\right) \in \mathscr{A}: x_{1} x_{2}=k\right\}\right| .
$$

Lemma 2.3. For any $d \mid P(z)$ with $d \leqslant \sqrt{D}$,

$$
\begin{aligned}
\sum_{k \equiv 0(\bmod d)} f(k)= & \frac{\pi}{16} \mathfrak{S}_{-}(m) \mathfrak{I}(m / N) N \prod_{p \mid d}\left(\frac{\omega_{1,0}(p)}{p}+\frac{\omega_{0,1}(p)}{p}-\frac{\omega_{1,1}(p)}{p^{2}}\right) \\
& +O\left(\sum_{\begin{array}{c}
d_{1}, d_{2}|d, d| d_{1} d_{2} \\
t_{1}\left|d / d_{1}, t_{2}\right| d d_{2} \\
\mathbf{d}=\left(d_{1} t_{1}, d_{2} t_{2}, 1,1\right)
\end{array}}|R(m, N, \mathbf{d})|\right) .
\end{aligned}
$$


Proof. Applying Lemma 2.2, we have

$$
\begin{aligned}
& \sum_{d \mid k} f(k)=\sum_{\substack{d_{1}, d_{2}|d \\
d| d_{1} d_{2}}}\left|\left\{\left(x_{1}, x_{2}, x_{3}, x_{4}\right) \in \mathscr{A}:\left(x_{1}, d\right)=d_{1},\left(x_{2}, d\right)=d_{2}\right\}\right| \\
& =\sum_{\substack{d_{1}, d_{2}\left|d\left(x_{1}, x_{2}, x_{3}, x_{4}\right) \in \mathscr{A} \\
d\right| d_{1} d_{2} \\
d_{1}\left|x_{1}, d_{2}\right| x_{2}}}\left(\sum_{t_{1} \mid\left(x_{1}, d\right) / d_{1}} \mu\left(t_{1}\right)\right)\left(\sum_{t_{2} \mid\left(x_{2}, d\right) / d_{2}} \mu\left(t_{2}\right)\right) \\
& =\sum_{\substack{d_{1}, d_{2}|d \\
d| d_{1} d_{2}\left|d_{1}\right| d / d_{1} \\
t_{2} \mid d / d_{2}}} \mu\left(t_{1}\right) \mu\left(t_{2}\right) \sum_{\begin{array}{c}
\left(x_{1}, x_{2}, x_{3}, x_{4}\right) \in \mathscr{A} \\
d_{1} t_{1}\left|x_{1}, d_{2} t_{2}\right| x_{2}
\end{array}} 1 \\
& =\sum_{\substack{d_{1}, d_{2}|d, d| d_{1} d_{2} \\
t_{1}\left|d / d_{1}, t_{2}\right| d / d_{2} \\
\mathbf{d}=\left(d_{1} t_{1}, d_{2} t_{2}, 1,1\right)}} \mu\left(t_{1}\right) \mu\left(t_{2}\right)\left(\frac{\omega(\mathbf{d}, m)}{d_{1} t_{1} d_{2} t_{2}} \frac{\pi}{16} \mathfrak{S}_{-}(m) \mathfrak{I}(m / N) N+R(m, N, \mathbf{d})\right) .
\end{aligned}
$$

In view of Lemma 2.1,

$$
\begin{aligned}
& \sum_{\substack{d_{1}, d_{2}|d, d| d\left|d_{1} d_{2} \\
t_{1}\right| d / d_{1}, t_{2} \mid d / d_{2} \\
\mathbf{d}=\left(d_{1} t_{1}, d_{2} t_{2}, 1,1\right)}} \mu\left(t_{1}\right) \mu\left(t_{2}\right) \frac{\omega(\mathbf{d}, m)}{d_{1} t_{1} d_{2} t_{2}} \\
= & \sum_{\substack{\left[d_{1}, d_{2}\right]=d \\
t_{1}\left|d_{2} /\left(d_{1}, d_{2}\right) \\
t_{2}\right| d_{1} /\left(d_{1}, d_{2}\right)}} \mu\left(t_{1}\right) \mu\left(t_{2}\right) \prod_{p \mid t_{1} t_{2}\left(d_{1}, d_{2}\right)} \frac{\omega_{1,1}(p)}{p^{2}} \prod_{p \mid d_{1} /\left(d_{1}, d_{2} t_{2}\right)} \frac{\omega_{1,0}(p)}{p} \prod_{p \mid d_{2} /\left(d_{1} t_{1}, d_{2}\right)} \frac{\omega_{0,1}(p)}{p} \\
= & \sum_{t_{1} t_{2} t_{3} t_{4} t_{5}=d} \mu\left(t_{1}\right) \mu\left(t_{2}\right) \prod_{p \mid t_{1} t_{2} t_{3}} \frac{\omega_{1,1}(p)}{p^{2}} \prod_{p \mid t_{4}} \frac{\omega_{1,0}(p)}{p} \prod_{p \mid t_{5}} \frac{\omega_{0,1}(p)}{p} \\
= & \prod_{p \mid d}\left(\frac{\omega_{1,0}(p)}{p}+\frac{\omega_{0,1}(p)}{p}-\frac{\omega_{1,1}(p)}{p^{2}}\right)
\end{aligned}
$$

Suppose that $0<m \leqslant 2 N$. Clearly,

$$
\begin{aligned}
& \left|\left\{\left(x_{1}, x_{2}, x_{3}, x_{4}\right): x_{1}^{2}+x_{4}^{2}=x_{2}^{2}+x_{3}^{2}+m, 1 \leqslant x_{i}^{2} \leqslant N,\left(x_{1} x_{2}, P(z)\right)=1\right\}\right| \\
& =\sum_{(k, P(z))=1} f(k) \leqslant \sum_{k} f(k)\left(\sum_{d \mid(k, P(z))} \lambda_{d}\right)^{2} \\
& =\sum_{d_{1}, d_{2} \mid P(z)} \lambda_{d_{1}} \lambda_{d_{2}} \sum_{k \equiv 0 \bmod \left[d_{1}, d_{2}\right]} f(k),
\end{aligned}
$$

where $\lambda_{d}$ are the weights appearing in Selberg's sieve method with $\lambda_{d}=0$ for $d \geqslant z$ (cf. [19, Chapter 3]). In view of Lemma 2.3 


$$
\begin{aligned}
& \sum_{d_{1}, d_{2} \mid P(z)} \lambda_{d_{1}} \lambda_{d_{2}} \sum_{k \equiv 0 \bmod \left[d_{1}, d_{2}\right]} f(k) \\
& =\frac{\pi}{16} \mathfrak{S}_{-}(m) \mathfrak{I}(m / N) N \sum_{d_{1}, d_{2} \mid P(z)} \lambda_{d_{1}} \lambda_{d_{2}} \prod_{p \mid\left[d_{1}, d_{2}\right]}\left(\frac{\omega_{1,0}(p)}{p}+\frac{\omega_{0,1}(p)}{p}-\frac{\omega_{1,1}(p)}{p^{2}}\right) \\
& +\sum_{d_{1}, d_{2} \mid P(z)} \lambda_{d_{1}} \lambda_{d_{2}} \cdot O\left(\sum_{\substack{d_{1}^{\prime}, d_{2}^{\prime}\left|\left[d_{1}, d_{2}\right],\left[d_{1}, d_{2}\right]\right| d_{1}^{\prime} d_{2}^{\prime} \\
t_{1}\left|\left[d_{1}, d_{2}\right] / d_{1}^{\prime}, t_{2}\right|\left[d_{1}, d_{2}\right] / d_{2}^{\prime} \\
\mathbf{d}=\left(d_{1} t_{1}, d_{2} t_{2}, 1,1\right)}}|R(m, N, \mathbf{d})|\right) .
\end{aligned}
$$

By Selberg's sieve method, we have

$$
\sum_{d_{1}, d_{2} \mid P(z)} \lambda_{d_{1}} \lambda_{d_{2}} \prod_{p \mid\left[d_{1}, d_{2}\right]}\left(\frac{\omega_{1,0}(p)}{p}+\frac{\omega_{0,1}(p)}{p}-\frac{\omega_{1,1}(p)}{p^{2}}\right)=\frac{1}{G_{1}(z)}
$$

where

$$
G_{1}(z)=\sum_{\substack{d \mid P(z) \\ d<z}} \prod_{p \mid d} \frac{\omega_{1,0}(p) p^{-1}+\omega_{0,1}(p) p^{-1}-\omega_{1,1}(p) p^{-2}}{1-\omega_{1,0}(p) p^{-1}-\omega_{0,1}(p) p^{-1}+\omega_{1,1}(p) p^{-2}}
$$

Since $\left|\lambda_{d}\right| \leqslant 1$

$$
\begin{aligned}
& \sum_{d_{1}, d_{2} \mid P(z)} \lambda_{d_{1}} \lambda_{d_{2}} \cdot O\left(\sum_{\substack{d_{1}^{\prime}, d_{2}^{\prime}\left|\left[d_{1}, d_{2}\right],\left[d_{1}, d_{2}\right]\right| d_{1}^{\prime} d_{2}^{\prime} \\
t_{1}\left|\left[d_{1}, d_{2}\right] / d_{1}^{\prime}, t_{2}\right|\left[d_{1}, d_{2}\right] / d_{2}^{\prime} \\
\mathbf{d}=\left(d_{1}, d_{2}\right.}}|R(m, N, \mathbf{d})|\right) \\
& \ll \sum_{\substack{d_{1}, d_{2} \mid P(z) \\
d_{1}, d_{2}<z^{2} \\
\mathbf{d}=\left(d_{1}, d_{2}, 1,1\right)}} \tau\left(d_{1}\right)^{2} \tau\left(d_{2}\right)^{2} \tau\left(d_{1} d_{2}\right)^{2}|R(m, N, \mathbf{d})| \ll N^{1-\epsilon / 2},
\end{aligned}
$$

where $\tau$ is the divisor function. Noting that $\omega_{1,0}(p), \omega_{0,1}(p)=1+O(1 / p)$ and $\omega_{1,1}(p)=O(1)$, we have (cf. [19, Lemma 4.1])

$$
G_{1}(z) \gg \prod_{p<z}\left(1+\frac{\omega_{1,0}(p)}{p}+\frac{\omega_{0,1}(p)}{p}-\frac{\omega_{1,1}(p)}{p^{2}}\right) \gg(\log z)^{2}
$$

Also, it had been shown [22, eq. (2.9)] that

$$
\mathfrak{S}_{-}(m) \ll \prod_{\substack{\beta \\ p^{\beta} \| m \\ p \geqslant 3 \\ \beta \geqslant 0}}\left(1+\frac{1}{p}-\frac{1}{p^{\beta+1}}-\frac{1}{p^{\beta+2}}\right)
$$


Finally,

$$
\begin{aligned}
& \mid\left\{\left(x_{1}, x_{2}, x_{3}, x_{4}\right) \in \mathscr{A}:\left|x_{1}\right|<z \text { or }\left|x_{2}\right|<z\right\} \mid \\
& \leqslant \sum_{\substack{\left|x_{1}\right|<z, x_{2}^{2} \leqslant N \\
\text { or }\left|x_{2}\right|<z, x_{1}^{2} \leqslant N}}\left|\left\{\left(x_{3}, x_{4}\right): x_{4}^{2}-x_{3}^{2}=x_{2}^{2}-x_{1}^{2}+m, x_{3}^{2}, x_{4}^{2} \leqslant N\right\}\right| \\
& \leqslant\left|\left\{\left(x_{1}, x_{2}\right):\left(x_{1}-x_{2}\right)\left(x_{1}+x_{2}\right)=m\right\}\right| \cdot\left|\left\{\left(x_{3}, x_{4}\right): x_{4}^{2}=x_{3}^{2}, x_{3}^{2}, x_{4}^{2} \leqslant N\right\}\right| \\
& +\sum_{\left|x_{1}\right|<z, x_{2}^{2} \leqslant N}\left|\left\{\left(x_{3}, x_{4}\right):\left(x_{4}-x_{3}\right)\left(x_{4}+x_{3}\right)=x_{2}^{2}-x_{1}^{2}+m\right\}\right| \\
& \text { or }\left|x_{2}\right|<z, x_{1}^{2} \leqslant N \\
& x_{2}^{2}-x_{1}^{2}+m \neq 0 \\
& \ll \tau(m) \sqrt{N}+\sum_{\substack{\left|x_{1}\right|<z, x_{2}^{2} \leqslant N \\
\text { or }\left|x_{2}\right|<z, x_{1}^{2} \leqslant N \\
x_{2}^{2}-x_{1}^{2}+m \neq 0}} \tau\left(x_{2}^{2}-x_{1}^{2}+m\right) \ll N^{2 / 3} z .
\end{aligned}
$$

Thus we obtain that

Theorem 2.1. For a positive integer $m$, we have

$$
\begin{aligned}
& \left|\left\{\left(p_{1}, p_{2}, b_{3}, b_{4}\right): p_{1}^{2}+b_{4}^{2}=p_{2}^{2}+b_{3}^{2}+m, p_{i} \in \mathcal{P}, b_{i} \in \mathbb{N}, p_{i}^{2}, b_{i}^{2} \leqslant N\right\}\right| \\
& \ll \frac{N}{(\log N)^{2}} \prod_{p \mid m}\left(1+\frac{1}{p}\right) .
\end{aligned}
$$

Now we are ready to prove Theorem 1.1 .

Proof of Theorem 1.1. Define

$$
r(x)=\left|\left\{(p, b, n): p^{2}+b^{2}+2^{n}=x, p \in \mathcal{P}, b, n \in \mathbb{N}\right\}\right| .
$$

Recall that $\mathcal{S}_{3}=\{x \in \mathbb{N}: r(x) \geqslant 1\}$. Then, by the Cauchy-Schwarz inequality,

$$
\begin{aligned}
\mid\{(p, b, n) & \left.: p^{2}+b^{2}+2^{n} \leqslant N, p \in \mathcal{P}, b, n \in \mathbb{N}\right\} \mid \\
& =\sum_{x \leqslant N} r(x) \leqslant \sqrt{\left|\mathcal{S}_{3} \cap[1, N]\right|} \cdot \sqrt{\sum_{x \leqslant N} r(x)^{2}} .
\end{aligned}
$$

Clearly,

$$
\begin{aligned}
& \left|\left\{(p, b, n): p^{2}+b^{2}+2^{n} \leqslant N, p \in \mathcal{P}, b, n \in \mathbb{N}\right\}\right| \\
& \quad \geqslant\left|\left\{p \in \mathcal{P}: p^{2} \leqslant N / 3\right\}\right| \cdot\left|\left\{b \in \mathbb{N}: b^{2} \leqslant N / 3\right\}\right| \cdot\left|\left\{n \in \mathbb{N}: 2^{n} \leqslant N / 3\right\}\right| \gg N .
\end{aligned}
$$

So it suffices to show that

$$
\sum_{x \leqslant N} r(x)^{2} \ll N
$$


Apparently,

$$
\left|\left\{(p, b): p^{2}+b^{2} \leqslant N, p \in \mathcal{P}, b \in \mathbb{N}\right\}\right| \ll \frac{N}{\log N} .
$$

Also, Rieger had proved [24, (h 2)] that

$$
\left|\left\{\left(p_{1}, p_{2}, b_{1}, b_{2}\right): p_{1}^{2}+b_{1}^{2}=p_{2}^{2}+b_{2}^{2} \leqslant N, p_{1} \neq p_{2}, p_{1}, p_{2} \in \mathcal{P}, b_{1}, b_{2} \in \mathbb{N}\right\}\right| \ll \frac{N}{\log N}
$$

Thus applying Theorem 2.1, we have

$$
\begin{array}{rl}
\sum_{x \leqslant N} & r(x)^{2}=\mid\left\{\left(p_{1}, p_{2}, b_{1}, b_{2}, n_{1}, n_{2}\right): p_{1}^{2}+b_{1}^{2}+2^{n_{1}}\right. \\
= & \left.p_{2}^{2}+b_{2}^{2}+2^{n_{2}} \leqslant N, p_{i} \in \mathcal{P}, b_{i}, n_{i} \in \mathbb{N}\right\} \mid \\
\ll & \sum_{2^{n_{1}}<2^{n_{2}} \leqslant N} \mid\left\{\left(p_{1}, p_{2}, b_{1}, b_{2}\right): p_{1}^{2}+b_{1}^{2}-p_{2}^{2}-b_{2}^{2}\right. \\
= & \left.2^{n_{2}}-2^{n_{1}}, p_{i}^{2}, b_{i}^{2} \leqslant N, p_{i} \in \mathcal{P}, b_{i} \in \mathbb{N}\right\} \mid \\
& +\sum_{2^{n} \leqslant N}\left|\left\{\left(p_{1}, p_{2}, b_{1}, b_{2}\right): p_{1}^{2}+b_{1}^{2}=p_{2}^{2}+b_{2}^{2} \leqslant N-2^{n}, p_{i} \in \mathcal{P}, b_{i} \in \mathbb{N}\right\}\right| \\
\ll & \sum_{2^{n_{1}<2^{n_{2}} \leqslant N}} \frac{N}{(\log N)^{2}} \prod_{p \mid 2^{n_{2}}-2^{n_{1}}}\left(1+\frac{1}{p}\right)+\frac{N}{\log N} \cdot \frac{\log N}{\log 2} .
\end{array}
$$

By Romanoff's arguments [25] (or see [23, p. 203]), we know that

$$
\sum_{2^{n_{1}}<2^{n_{2}} \leqslant N} \prod_{p \mid 2^{n_{2}-n_{1}}-1}\left(1+\frac{1}{p}\right) \ll(\log N)^{2} .
$$

\section{Proof of Theorems 1.2 and 1.3}

For an integer $a$ and a positive integer $n$, let $a(n)$ denote the residue class $\{x \in$ $\mathbb{Z}: x \equiv a(\bmod n)\}$. For a finite system $\mathcal{A}=\left\{a_{s}\left(n_{s}\right)\right\}_{s=1}^{k}$, we say $\mathcal{A}$ is a cover of $\mathbb{Z}$ provided that

$$
\bigcup_{s=1}^{k} a_{s}\left(n_{s}\right)=\mathbb{Z} .
$$

Our aim is to find a cover $\left\{a_{s}\left(n_{s}\right)\right\}_{s=1}^{k}$ of $\mathbb{Z}$ and distinct primes $p_{1}, p_{2}, \ldots, p_{k}$ with $p_{s} \equiv 3(\bmod 4)$ and $2^{n_{s}} \equiv 1\left(\bmod p_{s}\right)$. With help from the book [1], the following lemma can be directly verified. 
Lemma 3.1. Let

$$
\begin{aligned}
\left\{\left(a_{s}^{\prime}, n_{s}^{\prime}, p_{s}\right)\right\}_{s=1}^{49} & =\{(0,3,7),(1,15,11),(4,15,31),(7,15,151),(10,15,331), \\
& (13,105,43),(28,105,71),(43,105,127),(58,105,211), \\
& (73,105,29191),(88,105,86171),(103,315,870031), \\
& (208,315,983431),(313,315,1765891),(2,9,19),(5,27,87211), \\
& (14,81,71119),(41,81,97685839),(68,81,163),(23,135,271), \\
& (50,135,631),(77,135,811),(104,135,23311),(131,135,348031), \\
& (8,99,23),(17,99,67),(26,99,199),(35,99,683),(44,99,5347), \\
& (53,99,599479),(62,99,33057806959),(71,99,242099935645987), \\
& (80,495,991),(179,495,2971),(278,495,3191), \\
& (377,495,48912491),(476,495,2252127523412251),(89,693,463), \\
& (188,693,5419),(287,693,14323),(386,693,289511839), \\
& (485,693,35532364099),(584,693,2868251407519807), \\
& (683,693,581283643249112959),(98,297,694387), \\
& (197,297,14973866897175265228063698945547),(296,891,1783), \\
& (593,891,1409033313878253109224688819), \\
& (890,891,12430037668834128259094186647)\}
\end{aligned}
$$

Then $\mathcal{A}^{\prime}=\left\{a_{s}^{\prime}\left(n_{s}^{\prime}\right)\right\}_{s=1}^{49}$ is a cover of $\mathbb{Z}$. Furthermore, for $1 \leqslant s \leqslant 49$, we have $p_{s} \mid 2^{n_{s}^{\prime}}-1$ or $p_{s} \mid 2^{n_{s}^{\prime}}+1$.

Remark. In [29, Wu and Sun constructed a cover of $\mathbb{Z}$ with 173 odd moduli and distinct primitive prime divisors.

For $1 \leqslant s \leqslant 49$, let $n_{s}=2 n_{s}^{\prime}$ and let $a_{s}$ be an integer such that $a_{s} \equiv a_{s}^{\prime}\left(\bmod n_{s}^{\prime}\right)$ and $a_{s} \equiv 1(\bmod 2)$. Let $a_{50}=0, n_{50}=2$ and $p_{50}=3$. Then by the Chinese remainder theorem,

$$
\mathcal{A}=\left\{a_{s}\left(n_{s}\right)\right\}_{s=1}^{50}
$$

is a cover of $\mathbb{Z}$, and $2^{n_{s}} \equiv 1\left(\bmod p_{s}\right)$ for $1 \leqslant s \leqslant 50$. Let

$$
M_{1}=\prod_{s=1}^{50} p_{s}
$$

and let $\alpha_{1}$ be an integer such that

$$
\alpha_{1} \equiv 2^{a_{s}}\left(\bmod p_{s}\right)
$$

for $1 \leqslant s \leqslant 50$.

Let $x$ be an arbitrary positive integer with $x \equiv \alpha_{1}\left(\bmod M_{1}\right)$. Suppose that $x \in \mathcal{S}_{3}$, i.e., $x=p^{2}+b^{2}+2^{n}$ for some $p \in \mathcal{P}$ and $b, n \in \mathbb{N}$. Since $\mathcal{A}$ is a cover of $\mathbb{Z}$, there exists $1 \leqslant s \leqslant 50$ such that $n \equiv a_{s}\left(\bmod n_{s}\right)$. Then

$$
p^{2}+b^{2}=x-2^{n} \equiv \alpha_{1}-2^{a_{s}} \equiv 0\left(\bmod p_{s}\right) .
$$


Noting that $p_{s} \equiv 3(\bmod 4),-1$ is a quadratic nonresidue modulo $p_{s}$. It follows that

$$
p \equiv b \equiv 0\left(\bmod p_{s}\right)
$$

Since $p$ is prime, we must have $p=p_{s}$.

Below, we require some additional congruences. Arbitrarily choose distinct primes $q_{1}, q_{2}, \ldots, q_{50}$ such that $\left(q_{s}, M_{1}\right)=1$ and $q_{s} \equiv 7(\bmod 8)$ for $1 \leqslant s \leqslant 50$. Clearly, 2 is a quadratic residue and -1 is a quadratic non-residue modulo $q_{s}$. So $-2^{n}$ is a quadratic non-residue modulo $q_{s}$ for any $n \geqslant 0$. Let

$$
M_{2}=\prod_{s=1}^{50} q_{s}
$$

and let $\alpha_{2}$ be an integer such that

$$
\alpha_{2} \equiv p_{s}^{2}\left(\bmod q_{s}\right)
$$

for every $1 \leqslant s \leqslant 50$.

Let $M=M_{1} M_{2}$, and let $\alpha$ be an integer such that

$$
\alpha \equiv \alpha_{i}\left(\bmod M_{i}\right)
$$

for $i=1,2$. Then we have $\{x \in \mathbb{N}: x \equiv \alpha(\bmod M)\} \cap \mathcal{S}_{3}=\emptyset$. In fact, assume on the contrary, that $x \equiv \alpha(\bmod M)$ and $x=p^{2}+b^{2}+2^{n}$ for some $p \in \mathcal{P}$ and $b, n \in \mathbb{N}$. Noting that $x \equiv \alpha_{1}\left(\bmod M_{1}\right)$, we know $p=p_{s}$ for some $1 \leqslant s \leqslant 50$. But since $x \equiv \alpha_{2}\left(\bmod M_{2}\right)$,

$$
x-p_{s}^{2}-2^{n} \equiv \alpha_{2}-p_{s}^{2}-2^{n} \equiv-2^{n}\left(\bmod q_{s}\right) .
$$

So $x-p_{s}^{2}-2^{n}$ is a quadratic non-residue modulo $q_{s}$, which leads to an evident contradiction since $x-p_{s}^{2}-2^{n}=b^{2}$.

Now let us turn to the proof of Theorem 1.3. We still use the cover $\mathcal{A}$ in (函). Now suppose that $x \equiv-\alpha_{1}\left(\bmod M_{1}\right)$ and there exist $p \in \mathcal{P}$ and $b, n \in \mathbb{Z}$ such that $x=p^{2}+b^{2}-2^{n}$. Then $n \equiv a_{s}\left(\bmod n_{s}\right)$ for some $1 \leqslant s \leqslant 50$, and

$$
x+2^{n} \equiv-\alpha_{1}+2^{a_{s}} \equiv 0\left(\bmod p_{s}\right) .
$$

It follows that $p=p_{s}$. The main difficulty is to find the additional congruences. 


\section{Lemma 3.2. Let}

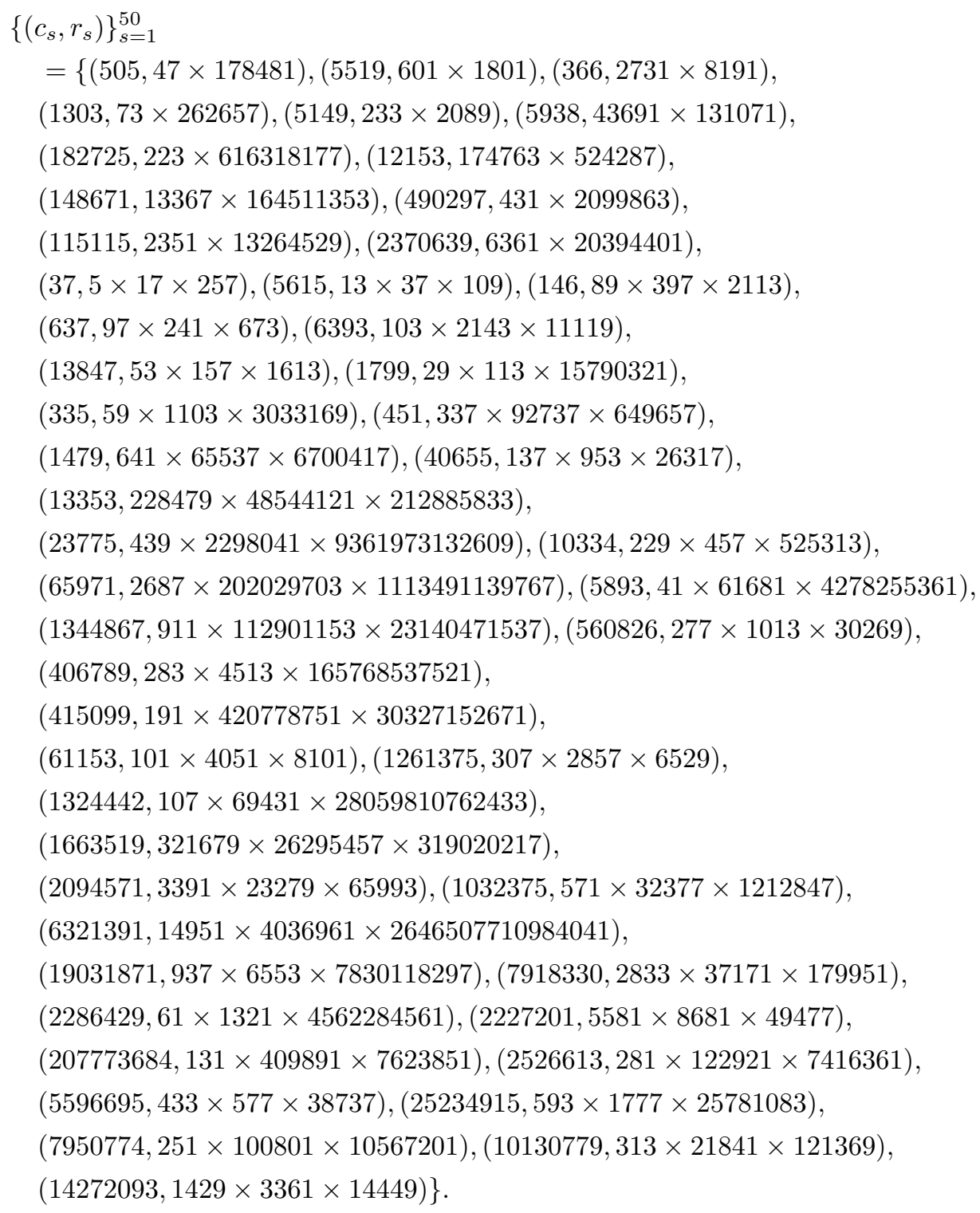

Then for every $1 \leqslant s \leqslant 50$ and $n \in \mathbb{N}, c_{s}+2^{n}$ is a quadratic non-residue modulo $r_{s}$.

Lemma 3.2 can be checked via a direct computation. In fact, we only need to consider those $c_{s}+2^{n}$ modulo $r_{s}$ for $0 \leqslant n<\operatorname{ord}_{2}\left(r_{s}\right)$, where $\operatorname{ord}_{2}(r)$ denotes the least positive integer such that $2^{\operatorname{ord}_{2}(r)} \equiv 1(\bmod r)$.

Notice that $\left(r_{i}, M_{1}\right)=1$ and $\left(r_{i}, r_{j}\right)=1$ for any distinct $i, j$. Let

$$
M_{3}=\prod_{s=1}^{50} r_{s}
$$

and let $\alpha_{3}$ be an integer such that

$$
\alpha_{3} \equiv c_{s}+p_{s}^{2}\left(\bmod r_{s}\right) .
$$


Let $M^{\prime}=M_{1} M_{3}$ and let $\alpha^{\prime}$ be an integer satisfying

$$
\alpha^{\prime} \equiv-\alpha_{1}\left(\bmod M_{1}\right) \quad \text { and } \quad \alpha^{\prime} \equiv \alpha_{3}\left(\bmod M_{3}\right) .
$$

For any $x \equiv \alpha^{\prime}\left(\bmod M^{\prime}\right)$, assume on the contrary that $x=p_{s}^{2}+b^{2}-2^{n}$ for some $1 \leqslant s \leqslant 50$ and $b, n \in \mathbb{N}$. Then

$$
b^{2}=x+2^{n}-p_{s}^{2} \equiv \alpha_{3}+2^{n}-p_{s}^{2} \equiv c_{s}+2^{n}\left(\bmod r_{s}\right) .
$$

This is impossible, since $c_{s}+2^{n}$ is a quadratic non-residue modulo $r_{s}$. Hence the residue class $\left\{x \in \mathbb{N}: x \equiv \alpha^{\prime}\left(\bmod M^{\prime}\right)\right\}$ contains no integer of the form $p^{2}+b^{2}-2^{n}$.

Remark. Observe that the moduli appearing in Lemma 3.2 are all composite. So we have the following problem.

Problem. Does there exist infinitely many primes $p$ such that the set

$\left\{1 \leqslant c \leqslant p: c+2^{n}\right.$ is a quadratic non-residue modulo $p$ for every $\left.n \in \mathbb{N}\right\}$

is non-empty?

In fact, we do not know of any such prime $p$. For example, let $p=2^{19}-1$, then $27006+2^{1}, 27006+2^{2}, \ldots, 27006+2^{18}$ are all quadratic non-residues modulo $p$, but $27006+2^{19}$ is a quadratic residue modulo $p$.

\section{The Integers OF THE FORM $b_{1}^{2}+b_{2}^{2}+2^{n^{2}}$}

Proof of the first assertion of Theorem 1.4. Let

$$
\mathcal{Q}=\{x \in \mathbb{N}: x \text { has no prime factor of the form } 4 k+3\} .
$$

Clearly,

$$
\mathcal{Q} \subseteq\left\{b_{1}^{2}+b_{2}^{2}: b_{1}, b_{2} \in \mathbb{N}\right\} .
$$

We only need to prove that the set

$$
\left\{x+2^{n^{2}}: x \in \mathcal{Q}, n \in \mathbb{N}\right\}
$$

has positive lower density. As an application of the half-dimensional sieve method [16], we know that

$$
|\{x \in \mathcal{Q}: x \leqslant N\}| \gg \frac{N}{\sqrt{\log N}} .
$$

With help from Selberg's sieve method, it is not difficult to see that

$$
\left|\left\{\left(x_{1}, x_{2}\right): x_{1}=x_{2}+m, x_{i} \in \mathcal{Q}, x_{i} \leqslant N\right\}\right| \ll \frac{N}{\log N} \prod_{\substack{p \mid m \\ p \equiv 3(\bmod 4)}}\left(1+\frac{1}{p}\right)
$$

for every positive integer $m$. By the Cauchy-Schwarz inequality,

$$
\begin{aligned}
& \left|\left\{x+2^{n^{2}}: x+2^{n^{2}} \leqslant N, x \in \mathcal{Q}, n \in \mathbb{N}\right\}\right| \\
& \quad \geqslant \frac{\left.\mid(x, n): x+2^{n^{2}} \leqslant N, x \in \mathcal{Q}, n \in \mathbb{N}\right\}\left.\right|^{2}}{\left|\left\{\left(x_{1}, x_{2}, n_{1}, n_{2}\right): x_{1}+2^{n_{1}^{2}}=x_{2}+2^{n_{2}^{2}} \leqslant N, x_{i} \in \mathcal{Q}, n_{i} \in \mathbb{N}\right\}\right|} .
\end{aligned}
$$

So it suffices to show that

$$
\left|\left\{\left(x_{1}, x_{2}, n_{1}, n_{2}\right): x_{1}+2^{n_{1}^{2}}=x_{2}+2^{n_{2}^{2}} \leqslant N, x_{i} \in \mathcal{Q}, n_{i} \in \mathbb{N}\right\}\right| \ll N .
$$


Now

$$
\begin{aligned}
& \left|\left\{\left(x_{1}, x_{2}, n_{1}, n_{2}\right): x_{1}+2^{n_{1}^{2}}=x_{2}+2^{n_{2}^{2}} \leqslant N, x_{i} \in \mathcal{Q}, n_{i} \in \mathbb{N}\right\}\right| \\
& \leqslant\left|\left\{\left(x_{1}, n_{1}\right): x_{1}+2^{n_{1}^{2}} \leqslant N, x_{1} \in \mathcal{Q}, n_{1} \in \mathbb{N}\right\}\right| \\
& +2 \sum_{0 \leqslant n_{1}<n_{2} \leqslant \sqrt{\log N / \log 2}}\left|\left\{\left(x_{1}, x_{2}\right): x_{1}-x_{2}=2^{n_{2}^{2}}-2^{n_{1}^{2}}, x_{i} \in \mathcal{Q} \cap[1, N]\right\}\right| \\
& \ll N+\frac{N}{\log N} \sum_{0 \leqslant n_{1}<n_{2} \leqslant \sqrt{\log N / \log 2}} \prod_{\substack{p \mid 2^{n_{2}^{2}}-2^{n_{1}^{2}} \\
p \equiv 3(\bmod 4)}}\left(1+\frac{1}{p}\right) .
\end{aligned}
$$

Obviously,

$$
\begin{aligned}
& \sum_{0 \leqslant n_{1}<n_{2} \leqslant \sqrt{\log N / \log 2}} \prod_{\substack{p \mid 2_{2}^{2}-2^{n_{1}^{2}} \\
p \equiv 3(\bmod 4)}}\left(1+\frac{1}{p}\right) \leqslant \sum_{0 \leqslant n_{1}<n_{2} \leqslant \sqrt{\log N / \log 2}} \prod_{p \mid 2^{n_{2}^{2}}-2^{n_{1}^{2}}}\left(1+\frac{1}{p}\right) \\
& \leqslant \sum_{d} \frac{1}{d} \sum_{\substack{0 \leqslant n_{1}<n_{2} \leqslant \sqrt{\log N / \log 2} \\
n_{2}^{2} \equiv n_{1}^{2}\left(\bmod \operatorname{ord}_{2}(d)\right)}} 1
\end{aligned}
$$

Suppose that $p$ is an odd prime, $\beta \geqslant 1$ and $1 \leqslant a \leqslant p^{\beta}$. Then we have

$$
\left|\left\{1 \leqslant x \leqslant p^{\beta}: x^{2} \equiv a\left(\bmod p^{\beta}\right)\right\}\right| \leqslant 2 p^{\nu_{p}(a) / 2},
$$

since the multiplicative group modulo $p^{\beta}$ is cyclic, where $\nu_{p}(a)$ denotes the greatest integer such that $p^{\nu_{p}(a)} \mid a$. Thus

$$
\sum_{\substack{0 \leqslant n_{1}<n_{2} \leqslant \sqrt{\log N / \log 2} \\ n_{2}^{2} \equiv n_{1}^{2}\left(\bmod \operatorname{ord}_{2}(d)\right)}} 1 \ll \sqrt{\frac{\log N}{\log 2}}\left(\sqrt{\frac{\log N}{\log 2}} \cdot \frac{2^{\omega\left(\operatorname{ord}_{2}(d)\right)} \sqrt{\operatorname{ord}_{2}(d)}}{\operatorname{ord}_{2}(d)}+1\right),
$$

where $\omega(r)$ denotes the number of distinct prime factors of $r$. We only need to prove that

$$
\sum_{d} \frac{2^{\omega\left(\operatorname{ord}_{2}(d)\right)}}{d \sqrt{\operatorname{ord}_{2}(d)}}
$$

converges. Define

$$
E(x)=\sum_{k \leqslant x} \sum_{\operatorname{ord}_{2}(d)=k} \frac{1}{d} .
$$

Romanoff had shown that $E(x) \ll \log x$ (cf. [23, pp. 200-201]). So

$$
\begin{aligned}
\sum_{d} \frac{2^{\omega\left(\operatorname{ord}_{2}(d)\right)}}{d \sqrt{\operatorname{ord}_{2}(d)}} & =\sum_{k} \frac{2^{\omega(k)}}{\sqrt{k}} \sum_{\operatorname{ord}_{2}(d)=k} \frac{1}{d} \\
& \ll \int_{1}^{\infty} x^{-1 / 3} d(E(x)) \\
& =\left.x^{-1 / 3} E(x)\right|_{1} ^{\infty}+\frac{1}{3} \int_{1}^{\infty} x^{-4 / 3} E(x) d x=O(1) .
\end{aligned}
$$




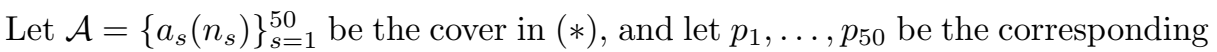
primes with $p_{s} \equiv 3(\bmod 4)$ and $p_{s} \mid 2^{n_{s}}-1$. Since every $p_{s}$ has at least one quadratic non-residue modulo $p_{s}$, the second assertion of Theorem 1.4 is an immediate consequence of the following stronger result.

Theorem 4.1. Let $\mathcal{N}$ be a set of non-negative integers. Suppose that for every $1 \leqslant s \leqslant 50$, there exists $1 \leqslant e_{s} \leqslant p_{s}$ such that

$$
\left|\mathcal{N} \cap\left\{x \in \mathbb{N}: x \equiv e_{s}\left(\bmod p_{s}\right)\right\}\right|<\infty .
$$

Then there exists a residue class with an odd modulus that contains no integer of the form $b_{1}^{2}+b_{2}^{2}+2^{n}$ with $n \in \mathcal{N}$.

Proof. Let $n_{s}^{*}=\operatorname{ord}_{2}\left(p_{s}\right)$. Noting that $n_{s}^{*} \mid n_{s}$ and $\left(n_{s}, p_{s}\right)=1$, for every $1 \leqslant s \leqslant$ 50 , let $a_{s}^{*}$ be an integer such that

$$
a_{s}^{*} \equiv a_{s}\left(\bmod n_{s}^{*}\right)
$$

and

$$
a_{s}^{*} \equiv e_{s}\left(\bmod p_{s}\right)
$$

Clearly, $\mathcal{A}^{*}=\left\{a_{s}^{*}\left(n_{s}^{*}\right)\right\}_{s=1}^{50}$ is also a cover of $\mathbb{Z}$.

Let

$$
\mathscr{H}_{s}=\mathcal{N} \cap\left\{x \in \mathbb{N}: x \equiv e_{s}\left(\bmod p_{s}\right)\right\}=\left\{h_{s, 1}, h_{s, 2}, \ldots, h_{s,\left|\mathscr{H}_{s}\right|}\right\}
$$

for $1 \leqslant s \leqslant 50$. Choose $\left|\mathscr{H}_{1}\right|+\left|\mathscr{H}_{2}\right|+\cdots+\left|\mathscr{H}_{50}\right|$ distinct primes

$$
q_{1,1}, \ldots, q_{1,\left|\mathscr{H}_{1}\right|}, q_{2,1}, \ldots, q_{2,\left|\mathscr{H}_{2}\right|}, \ldots, q_{50,1}, \ldots, q_{50,\left|\mathscr{H}_{50}\right|}
$$

satisfying

$$
q_{s, t} \equiv 3(\bmod 4)
$$

and

$$
q_{s, t} \notin\left\{p_{1}, p_{2}, \ldots, p_{50}\right\}
$$

for every $1 \leqslant s \leqslant 50$ and $1 \leqslant t \leqslant\left|\mathscr{H}_{s}\right|$.

Let

$$
M^{*}=\left(\prod_{1 \leqslant s \leqslant 50} p_{s} \cdot \prod_{\substack{1 \leqslant s \leqslant 50 \\ 1 \leqslant t \leqslant\left|\mathscr{H}_{s}\right|}} q_{s, t}\right)^{2}
$$

and $\alpha^{*}$ be an integer such that

$$
\alpha^{*} \equiv 2^{a_{s}^{*}}\left(\bmod p_{s}^{2}\right)
$$

and

for every $s, t$.

$$
\alpha^{*} \equiv 2^{h_{s, t}}+q_{s, t}\left(\bmod q_{s, t}^{2}\right)
$$

We claim that for any $x \equiv \alpha^{*}\left(\bmod M^{*}\right), x$ is not of the form $b_{1}^{2}+b_{2}^{2}+2^{n}$ with $n \in \mathcal{N}$. Assume on the contrary that $x \equiv \alpha^{*}\left(\bmod M^{*}\right)$ and $x=b_{1}^{2}+b_{2}^{2}+2^{n}$ with $n \in \mathcal{N}$. Since $\mathcal{A}^{*}=\left\{a_{s}^{*}\left(n_{s}^{*}\right)\right\}_{s=1}^{50}$ is a cover, by arguing similarly as in the proof of Theorems 1.2 and 1.3 , we know that

$$
b_{1} \equiv b_{2} \equiv 0\left(\bmod p_{s}\right)
$$

for some $1 \leqslant s \leqslant 50$. It follows that

$$
x-2^{n} \equiv 2^{a_{s}^{*}}-2^{n} \equiv 0\left(\bmod p_{s}^{2}\right),
$$


that is, $n \equiv a_{s}^{*}\left(\bmod \operatorname{ord}_{2}\left(p_{s}^{2}\right)\right)$. It is not difficult to check that

$$
2^{n_{s}^{*}}=2^{\operatorname{ord}_{2}\left(p_{s}\right)} \not \equiv 1\left(\bmod p_{s}^{2}\right) \text {. }
$$

(In fact, the only known primes $p$ with $2^{p-1} \equiv 1\left(\bmod p^{2}\right)$ are 1093 and 3511.) Also,

$$
2^{n_{s}^{*} p}=\sum_{k=0}^{p}\left(\begin{array}{l}
p \\
k
\end{array}\right)\left(2^{n_{s}^{*}}-1\right)^{k} \equiv 1\left(\bmod p_{s}^{2}\right) .
$$

So we must have $\operatorname{ord}_{2}\left(p_{s}^{2}\right)=n_{s}^{*} p$. Consequently,

$$
n \equiv a_{s}^{*} \equiv e_{s}\left(\bmod p_{s}\right) \text {. }
$$

Since $n \in \mathcal{N}$, we have $n \in \mathscr{H}_{s}$ and there exists $1 \leqslant t \leqslant\left|\mathscr{H}_{s}\right|$ such that $n=h_{s, t}$. It follows that

$$
b_{1}^{2}+b_{2}^{2}=x-2^{n} \equiv \alpha^{*}-2^{h_{s, t}} \equiv q_{s, t}\left(\bmod q_{s, t}^{2}\right) .
$$

So $b_{1}^{2}+b_{2}^{2} \equiv 0\left(\bmod q_{s, t}\right)$. However, recalling that $q_{s, t} \equiv 3(\bmod 4), q_{s, t}$ divides $b_{1}^{2}+b_{2}^{2}$ implies that

Hence,

$$
b_{1} \equiv b_{2} \equiv 0\left(\bmod q_{s, t}\right)
$$

$$
b_{1}^{2}+b_{2}^{2} \equiv 0\left(\bmod q_{s, t}^{2}\right),
$$

which is evidently contradicted with $(\diamond)$.

Corollary 4.1. There exists a positive integer $m$ such that the set

$$
\left\{x \in \mathbb{N}: x \text { is even and } x \text { is not of the form } b_{1}^{2}+b_{2}^{2}+2^{m n}\right\}
$$

contains an infinite arithmetic progression.

Proof. Let $m=p_{1} p_{2} \ldots p_{50}$ where $p_{1}, p_{2}, \ldots, p_{50}$ are the primes in Lemma 3.1. Thus substituting $\mathcal{N}=\{x \in \mathbb{N}: x \equiv 0(\bmod m)\}$ and $e_{s}=1$ in Theorem 4.1. we are done.

Problem. Does there exist a residue class with an odd modulus that contains no integer of the form $b_{1}^{2}+b_{2}^{2}+2^{n}$ with $b_{1}, b_{2}, n \in \mathbb{N}$ ?

\section{ACKNOWLEDGEMENTS}

We express our gratitude to the anonymous referee for his/her very useful comments on our paper. We are also grateful to Professors Hongze Li and Zhi-Wei Sun for their helpful suggestions.

\section{REFERENCES}

[1] J. Brillhart, D. H. Lehmer, J. L. Selfridge, B. Tuckerman, and S. S. Wagstaff, Jr., Factorizations of $b^{n} \pm 1, b=2,3,5,6,7,10,11,12$ up to High Powers, 3rd ed., Contemporary Mathematics 22, Amer. Math, Soc., Providence, RI, 2002.

[2] J. Brüdern and E. Fouvry, Lagrange's four squares theorem with almost prime variables, J. Reine Angew Math., 454(1994), 59-96. MR1288679(96e:11125)

[3] Y.-G. Chen, On integers of the form $2^{n} \pm p_{1}^{\alpha_{1}} \cdots p_{r}^{\alpha_{r}}$, Proc. Amer. Math. Soc., 128(2000), 1613-1616. MR.1695159 (2000j:11006)

[4] Y.-G. Chen, On integers of the form $k 2^{n}+1$, Proc. Amer. Math. Soc., 129(2001), 355-361. MR.1800230 (2003a:11004)

[5] Y.-G. Chen, On integers of the form $k-2^{n}$ and $k 2^{n}+1$, J. Number Theory, 89(2001), 121-125. MR:1838707 (2002b:11020)

[6] Y.-G. Chen, On integers of the forms $k^{r}+2^{n}$ and $k^{r} 2^{n}+1$, J. Number Theory, 98(2003), 310-319. MR 1955419 (2003m:11004) 
[7] Y.-G. Chen, Five consecutive positive odd numbers, none of which can be expressed as a sum of two prime powers, Math. Comp., 74(2005), 1025-1031. MR2114663(2006a:11010)

[8] Y.-G. Chen, On integers of the forms $k \pm 2^{n}$ and $k 2^{n} \pm 1$, J. Number Theory, 125(2007), 14-25. MR2333115 (2008e:11015)

[9] Y.-G. Chen, R. Feng and N. Templier, Fermat numbers and integers of the form $a^{k}+a^{l}+p^{\alpha}$, Acta Arith., 135(2008), 51-61. MR2453523 (2009h:11023)

[10] F. Cohen and J. L. Selfridge, Not every number is the sum or difference of two prime powers, Math. Comp., 29(1975), 79-81. MR0376583 (51:12758)

[11] R. Crocker, On the sum of a prime and two powers of two, Pacific J. Math., 36(1971), 103-107. MR0277467 (43:3200)

[12] R. Crocker, On the sum of two squares and two powers of $k$, Colloq. Math., 112(2008), 235-267. MR2383332 (2009a:11210)

[13] J. G. van der Corput, On de Polignac's conjecture, Simon Stevin, 27(1950), 99-105. MR0035298 (11:714e)

[14] P. Erdős, On integers of the form $2^{k}+p$ and some related problems, Summa Brasil. Math. 2(1950), 113-123. MR0044558 (13:437i)

[15] H. Iwaniec, Primes of the type $\phi(x, y)+A$ where $\phi$ is a quadratic form, Acta Arith., 21(1972), 203-234. MR0304331 (46:3466)

[16] H. Iwaniec, The half dimensional sieve, Acta Arith., 29(1976), 69-95. MR0412134 (54:261)

[17] H. Iwaniec, Rosser's sieve, Acta Arith., 36 (1980), 171-202. MR.581917 (81m:10086)

[18] H. Iwaniec, A new form of the error term in the linear sieve, Acta Arith., 37 (1980), 307-320. MR598883 (82d:10069)

[19] H. Halberstam and H.-E. Richert, Sieve methods, London Mathematical Society Monographs, 4, Academic Press, London-New York, 1974. MR0424730 (54:12689)

[20] E. Landau, Über die Einteilung der positiven ganzen Zahlen in vier Klassen nach der Mindeszahl der zu ihrer additiven Zusammensetzung erforderlichen Quadrate, Arch. Math. Phys., 13(1908), 305-312.

[21] F. Luca and P. Stănică, Fibonacci numbers that are not sums of two prime powers, Proc. Amer. Math. Soc., 133(2005), 1887-1890. MR2099413(2005k:11023)

[22] J.-Y. Liu, M.-C. Liu and T. Zhan, Squares of primes and powers of 2, Monatsh. Math., 128(1999), 283-313. MR 1726765 (2000k:11110)

[23] M. B. Nathanson, Additive Number Theory: The Classical Bases, Grad. Texts Math. 164, Springer-Verlag, New York, 1996. MR.1395371 (97e:11004)

[24] G. J. Rieger, Über die Summe aus einem Quadrat und einem Primzahlquadrat, J. Reine Angew Math., 231(1968), 89-100. MR0229603 (37:5177)

[25] N. Romanoff, Über einige Sätze der additiven Zahlentheorie, Math. Ann., 109(1934), 668678. MR 1512916

[26] Z. W. Sun, On integers not of the form $\pm p^{a} \pm q^{b}$, Proc. Amer. Math. Soc., 128(2000), 997-1002. MR1695111 (2000i:11157)

[27] Z.-W. Sun and M.-H. Le, Integers not of the form $c\left(2^{a}+2^{b}\right)+p^{\alpha}$, Acta Arith., 99(2001), 183-190. MR1847620 (2002e:11043)

[28] Z.-W. Sun and S.-M. Yang, A note on integers of the form $2^{n}+c p$, Proc. Edinburgh Math. Soc., 45(2002), 155-160. MR1884609 (2002j:11117)

[29] K.-J. Wu and Z.-W. Sun, Covers of the integers with odd moduli and their applications to the forms $x^{m}-2^{n}$ and $x^{2}-F_{3 n} / 2$, Math. Comp., 78(2009), 1853-1866. MR 2501080 (2010g:11019)

[30] P. Z. Yuan, Integers not of the form $c\left(2^{a}+2^{b}\right)+p^{\alpha}$, Acta Arith., 115(2004), 23-28. MR2102803 (2005f:11035)

Department of Mathematics, Nanjing University, Nanjing 210093, People's Republic OF CHINA

E-mail address: haopan79@yahoo.com.cn

Department of Mathematics, Nanjing University, Nanjing 210093, People's Republic OF CHINA

E-mail address: zhangwei_07@yahoo.com.cn 